\title{
Beyond the White Cube: Presentation of Visual Art in Interactive 3D Environments
}

\author{
Jeni Maleshkova \\ Queen Mary University of London \\ Media and Arts Technology DTC \\ School of Electronic Engineering \\ and Computer Science \\ United Kingdom \\ j.maleshkova@qmul.ac.uk
}

\author{
Matthew Purver \\ Queen Mary University of London \\ Cognitive Science Research Group \\ School of Electronic Engineering \\ and Computer Science \\ United Kingdom \\ m.purver@qmul.ac.uk
}

\begin{abstract}
New Media has not only revolutionized the way art is made but also how it is presented and curated. In this context, Virtual Reality offers exciting new possibilities, which can be encapsulated as 3D interactive features for the Web. We present two projects along these lines, and describe their effects on user engagement and interaction.
\end{abstract}

Art presentation. Art communication. Interaction. Virtual reality. Curating.

\section{INTRODUCTION}

The challenge of modern exhibitions is to invite the viewer not just to stand and stare but also to think along. Digital technologies can help this, by providing an escape from reality, offering the option to view things in an imaginary world or in ways that the physical world does not allow. The majority of current art display applications, however, aim to look and feel like the real world; we take the opposite approach, aiming to exploit digital possibilities to offer a different experience from that the user has in a common exhibition space.

Following this framework, we present two examples which showcase how advanced technical tools can be applied for the presentation of visual art. Both projects contribute towards escaping from the common exhibition space, giving the user the option to explore paintings not only interactively but also in a unique surrounding, difficult to simulate in the real world. First, we propose a Virtual Reality pavilion, whose design purpose is to contribute to the perception of the displayed paintings. Second, we provide a free-viewpoint video implementation for the Web, creating a common place where portraits and real actors perform together in a virtual environment as players in a storytelling presentation, and where the user can interactively explore the artworks and connections between them.

For the second project, two user studies have been performed. The first study compares our virtual environment approach with that of a conventional
3D art gallery. Both virtual exhibitions have identical visual and text information, delivered in two different ways - as a typical virtual gallery and using our interactive application. The results show that the proposed approach was perceived as more enjoyable and engaging. The second user study took place at the Victoria and Albert Museum in London. There the project was presented as an interactive installation with which visitors were able to explore the paintings.

\section{MOTIVATION}

Only until recently, the traditional view of the framework for art and media presentation was guided by commonly accepted and fixed stereotypes. The presentation of art to a broad audience began with the Parisian salons in the late baroque time. In these salons paintings were displayed on the whole wall without any concept of placement and just following the rule - large paintings on the top, small ones on the bottom. Later on in the 19th century artworks were usually presented only on eye level. Since the $20^{\text {th }}$ century the ideal room for exhibiting visual art is believed to not overwhelm the artwork and it should be as simple as possible (O'Doherty 1986). The artists of the 20th century were used of having white surrounding as an exhibition space. Usually this environment was taken as a given, without offering much option for changing or adjusting it in accordance with the presented artwork. Similarly, modern museums were designed and conceptualised in such a way that the inner space 
offered very few options for adjustments and tailoring to the particular artist's need.

The use of new technologies in the field of art presentation gives artist and curators numerous exciting opportunities. Beryl Graham states in his book 'Rethinking Curating - Art after New Media' (Graham 2010):

\begin{abstract}
"New media art presents the opportunity for a complete rethink of curatorial practice, from how art is legitimated and how museum departments are founded to how curators engage with the production of artwork and how they set about the many tasks within the process of showing that art to an audience."
\end{abstract}

The innovation potential in the context of media presentation and exhibition spaces design is very high. The viewer of modern exhibitions is not invited to just stand and observe, but to think along. In the context of employing new technologies for art display, Virtual Reality and the benefits it offers can be very useful. Furthermore, these can be mirrored in the form 3D features for websites, which can be used for communication and presentation of art.

In this context, the projects presented here aim to contribute towards escaping from the common space for art presentation by creating a virtual application that benefits from the technology used and augments the presented artwork by offering an interactive and enhanced audience experience. It is not our intention that the virtual space should look and feel like the real world, which the majority of the current applications are aiming to achieve, but rather provide an escape from reality, offering the option to view and explore things in a simulated world and to create knowledge about the artworks of interest. Therefore, our purpose is to offer a different experience from that the user has in a common exhibition space.

\section{RELATED WORK}

An early study discussing approaches of creating an Internet gallery is presented in (Muller et al. 1999). The authors argue that the most important aspect of an Internet gallery is visualisation, while the physical presence has just a secondary significance, especially for the buying process. Following this concept the main characteristics of the virtual gallery are defined to be fast search functionality and displaying of the user's query result in good quality. Later papers (Hendricks et al. 2003, Ullrich et al. 2002) emphasize the importance of 3D presentation and interactive navigation in virtual museum and galleries.

One of the central findings is that visitors in galleries, organized as 3D environments with higher level of navigational support, are more likely to explore the gallery and enjoy themselves in comparison to other galleries. A recent work (Fassbender et al. 2010) considers the opportunities of involving three new media technologies into art galleries in Australia to serve remote communities. The discussed technologies comprise: user-controlled Internet camera, livestream of point-of-view curator floor talks and a Second Life art gallery with voice/text chat. The results support the thesis that introducing usercontrolled devices significantly increase the time spent viewing paintings. Methods for developing an embodied tour guide through avatars are described in Xiang et al. (2003) and Zheng et al. (2005).

Introducing new methods and technologies in virtual art museums and galleries can be considered in several directions. One group of researchers (Amicis et al. 2009, Cavazza et al. 2001 ) investigate the prospective of involving game engine's technology. These tools can be incorporated both: in the design of the spatial layout of the virtual gallery and in editing and manipulating high-quality textures representing portions of the original images. Another trend for involving haptic devices in the virtual reality system and providing so the user with the ability to interact with the environment not only through the senses of sight and hearing but also of touch is presented by (Bergamasco et al. 2002 and Minamizawa et al. 2012). Concerning the description of the virtual scene it can be noticed that the Virtual Reality Modelling Language (VRML) is still dominant in most implementations (Eckert et al. 2010, Fortino et al. 2002, Hernandez et al. 2003, Masa et al. 2003). The traditional functions of a virtual gallery to visualize virtual objects are enhanced in each of this works by complementary features like movie on-demand, wireless multiuser communication, and social networking.

Presentation of new media art projects is given in (Bovcon 2008, Hung et al. 2010, Singh 2011). These papers make well-known artists and their work popular to the computer graphics research community and demonstrate potential fields of artistic and creative applications.

\section{OUR APPROACH}

To our understanding above and beyond technical improvements, a critical issue for further development is the creation of new approaches and methods for delivering the content to the user in an involving and entertaining way. The virtual environment should not try to reproduce the real one. Instead the virtual reality system has to create a specific environment, a space where the viewer 
gets additional information about the artworks and can explore and discover new facts and details.

We aim to empower the user to interactively explore the virtual exhibition space, without having to physically go somewhere. Our goal is to develop an application that gives the user the opportunity to explore artworks interactively in a virtual environment. Furthermore, the intention here is to focus on the advantages that 3D technology offers for viewing and exploring paintings in a virtual space and not to try to recreate a completely realistic environment, as we know it from common exhibition spaces. We want to leave behind the usual gallery surrounding for presentation of artworks and offer the viewers the possibility to discover and interpret the paintings on their own. We want to design a system that allows people to interactively explore visual art.

Furthermore, we want to engage the users by involving them into a story that connects the represented paintings. Our intention is to deliver the information about the paintings not just as a list of facts but as a narrative that stimulates people to think along and feel more engaged with the art.

\section{PROJECTS}

New media artists are expanding their practice continually using increasingly complex technical tools. We present two projects, which explore not only the presentation of visual arts using the state of the art VR technologies, but also investigate the sense of escapism and journey, which audiences seek out when selecting to engage with viewing contemporary new media art in this medium. The aim of the first project is to design an artificial presentation space, which contributes to the creation of an appropriate atmosphere for better perception of the message the artwork is intended to deliver. We analyse not only how art works (such as paintings) in VR are observed and perceived, but also their relationship to the virtual surroundings they occupy and how the users of VR equipment navigate around a virtual space to view them. We do this first by creating a framework in which we design virtual pavilions in the traditional style of physical pavilions (like those which exist in the physical world), but also using the advantages of unique VR surroundings to which VR world designers and users are already accustomed. A deeper investigation is sought by a second project, which promotes interaction from observers through the use of a specially constructed common area in which actors perform in a virtual environment populated by distinguished and easily recognisable portraits in the virtual space. The intention here is to stimulate the viewer to step out of the role of passive spectator, to become an active participant and to discover some pieces of information hidden in the environment, gaining knowledge about the observed objects in an entertaining manner.

\subsection{Virtual reality pavilion}

In this project the arrangement of the visualisation environment is especially designed for Stefan Hoenerloh's pictures. The artist's work represents the architecture of an imaginary city, with views that are true to the reality. This city exists only in his creative mind. Exposed in an especially designed exhibition space, the paintings should increase their impact and the way they are observed. The viewer is confronted with an exhibition environment, divided into two vertical levels, which further considered, should represent two different worlds - one of Hoenerloh's abandoned city and another, more similar to ours, full of light and life. On the ground level, the visitor is welcomed in a bright space with green plants and comfortable seats (see Figure 1). On the upper level, a platform functions as a lookout stage for observation of the imaginary city. In the same time, standing on the platform the viewer can still see the ground level, which in contrast to the paintings, is full of life (Figure 2).

The possibilities of stereoscopic visualisation of the pavilion both on a desktop and on immersive VR systems have been investigated. This approach gives us an opportunity to design environments in which the artworks can fully expand their impact and the user has the possibility to have an interactive experience and explore the art in a different context.

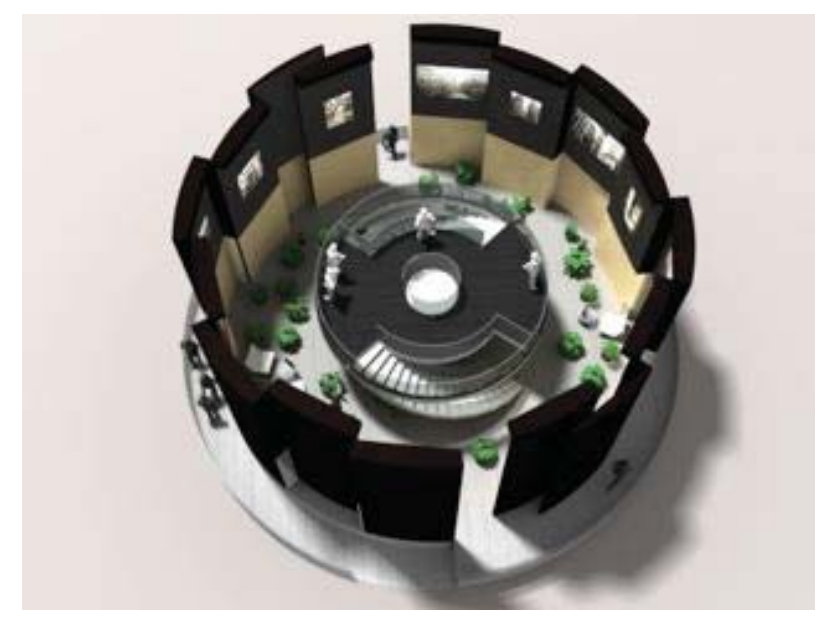

Figure 1: Image of the Virtual Reality Pavilion 


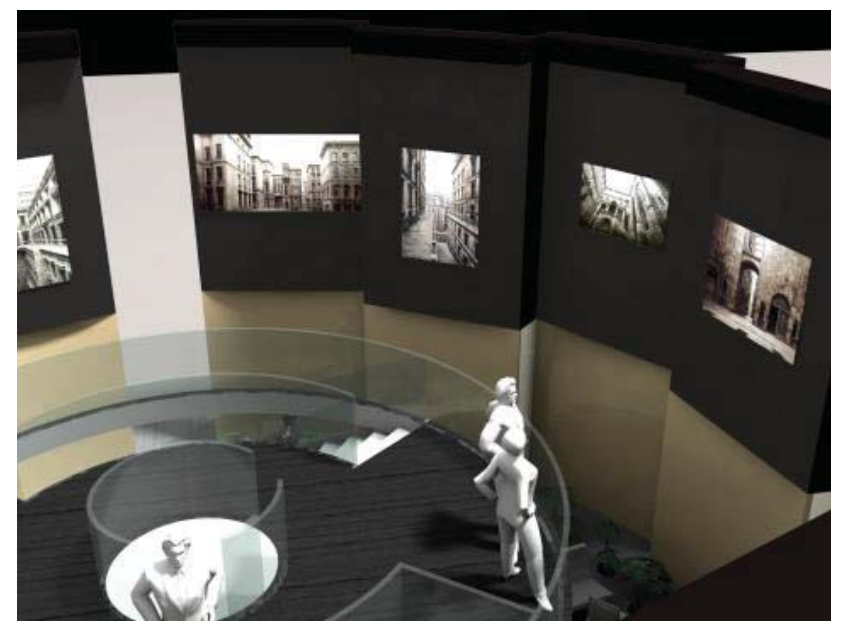

Figure 2: Image of the Virtual Reality Pavilion

\subsection{A WebGL implementation of a free- viewpoint video}

The second project was implemented using a multicamera capturing system and a 3D WebGL rendering tool (Maleshkova et al. 2012). The storyline for the shoot of the video is an actor who matches the pose of the character in each of five selected portraits. The actor changes his body posture according to the pose of the character in the corresponding painting. The portraits are chosen because they are all connected to one another and tell a story that the user is challenged to discover by navigating into the virtual space and looking for the artist perspective (Figure 3). Once the correct view point and angle are found the image of the portrait appears (Figure 4) along with some text information about the artist and the connection between the five paintings.

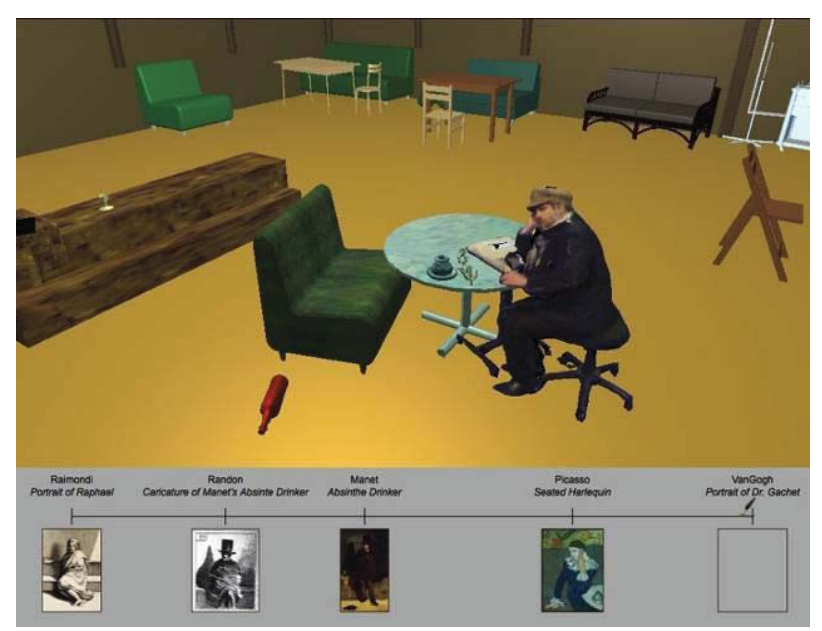

Figure 3: Top: the scene with the actor taking the pose of the character in the corresponding painting. Bottom: the storyline of the five portraits

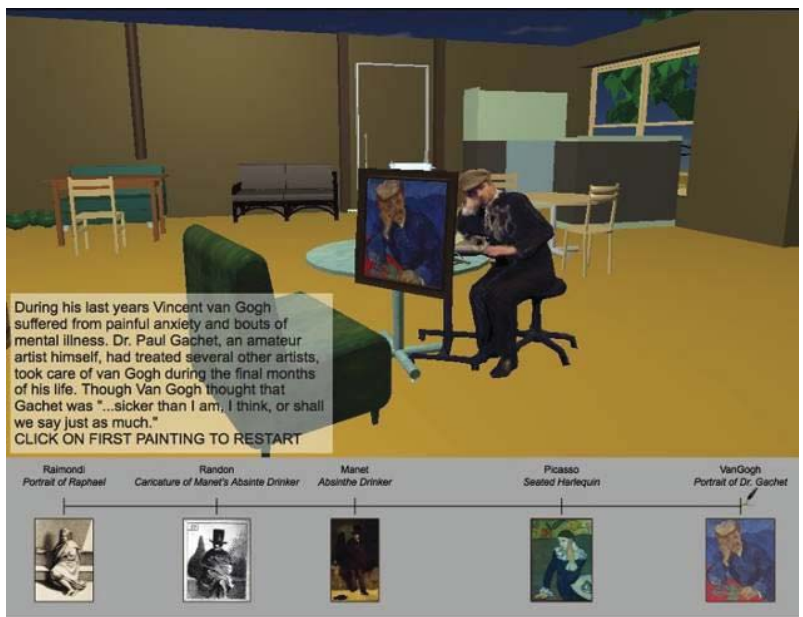

Figure 4: When the correct view point and angle are found the image of the portrait with accompanying information are shown

\section{STUDIES}

Two studies have been performed to investigate user behaviour and engagement with the second project.

\subsection{First study}

As part of the study two prototypes have been developed. Both of them display the same five portraits of well-known painters. However, the tools that are used to communicate the narrative behind these paintings are quite different. In the first prototype we have executed a rather innovative method using the free-viewpoint video capturing system, described in section 5.2.

Prototype two is implemented by using the game engine Unity. Here the paintings are displayed in a virtual environment, which recreates the setting of a typical gallery room (Figure 5). The interactivity of this prototype is limited to the navigation in the virtual room. The information to each portrait is shown as a text next to each image.

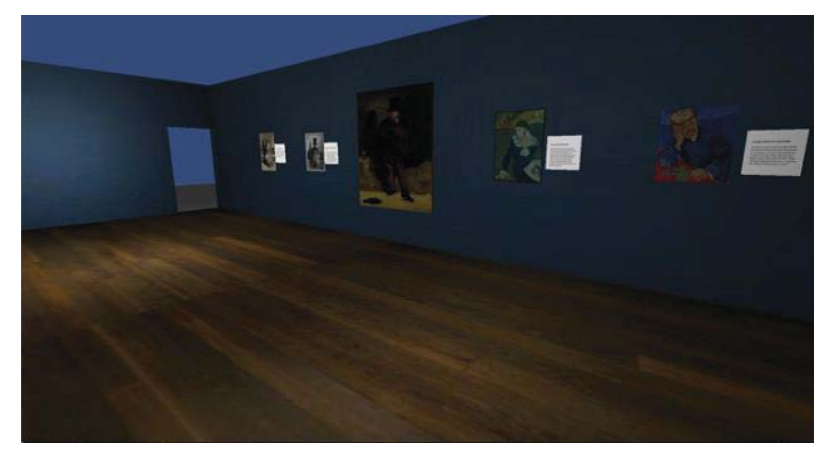

Figure 5: Paintings presented in a typical virtual gallery room 
This study aims to compare the two different approaches. We have two prototypes of 3D environments, which display the same five paintings and give identical text information but use diverse methods for communicating them. We want to examine the differences in the perception of the information delivered through two different approaches. The order in which the prototypes are shown to the participants is important for the analysis of the results of this study. Thus the participants were divided into two similar groups the first group was shown Prototype 1 first, then Prototype 2. To the second group the prototypes were presented vice versa. In order to have two fairly identical groups of people, the participants were requested to complete a short survey where they were asked to share general information about themselves such as name, gender, age and occupation. Additionally, to establish their level of knowledge in the fields of visual art and virtual reality technology, participants had to perform a small task online. The results from the survey were used to split the participants in two groups. However, in the process of the design of the study an additional survey was launched that asks people directly to rate their level of knowledge. From the first survey we assumed people's expertise from their answers, whereas in the second survey we took participant's own judgment for validation. Both surveys helped us to define the two groups but also gave us an insight of the overlap between people's self-evaluation and the results from their answers.

Once the online survey was completed, people were asked to take part in a one-to-one workshop session. Participants were encouraged to set their imagination free and present the five paintings in any possible way using everyday crafting materials (Figure 6). Users were given no information about the paintings. Instead, they were asked to make their own connections between the portraits and if they could - think about a story that might put the artworks in the same context. Participants were left to think for circa five to ten minutes about how and why would they present the paintings. Next, they were asked to make a sketch or a paper prototype of their display solution and explain the intention behind their concept (Figure 7). The display ideas from the paper prototypes were collected and are going to be integrated in user-initiated design features for a new virtual gallery prototype.

After the design task, the participants were shown the two prototypes. The task given to them was to explore the paintings in the two virtual environments. After experiencing both art presentation exhibition forms, people were asked for their feedback by using a questionnaire. They compared both prototypes on a scale according to their perception on level of engagement and information communication in both prototypes. The final part of the survey was to ask the users to define the two virtual exhibitions in a few words.

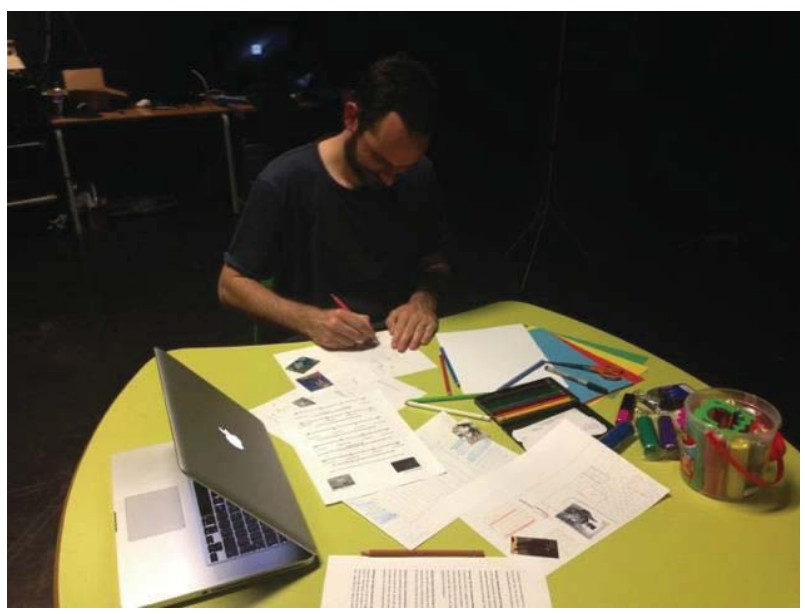

Figure 6: Users participating in the first study

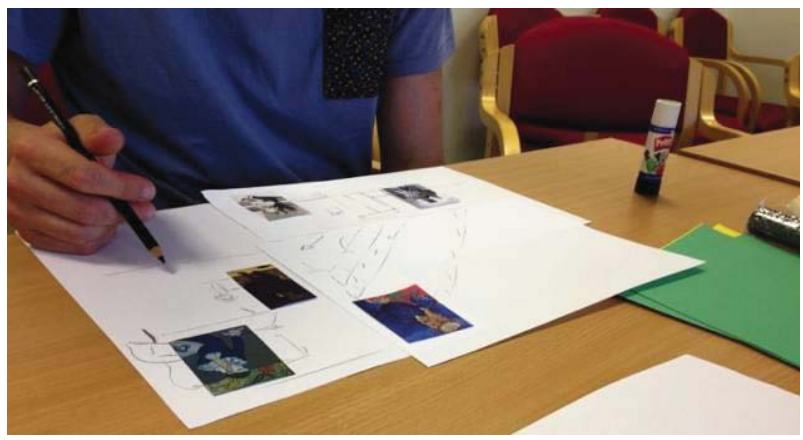

Figure 7: Users making a paper prototype

\subsection{Results from the first study}

The results from the first study can be summarised as follow:

- In both groups the prototype shown second was always considered as more informative and the story behind the paintings was better understood;

- Regardless of the order in which both virtual galleries were presented, participants felt more engaged with the narrative using the interactive video application;

- Participants in both groups found the interactive video application more enjoyable and fun to use;

- The interactive video application was described by the test users as a 'game', while the second prototype was defined as a 'virtual gallery';

- The ability to see all five paintings at once was positively rated in the virtual gallery version. 


\subsection{Second study}

The second study took place at the Victoria and Albert Museum in London. The project described in section 5.2 was exhibited as in interactive installation in the scope of a monthly event called 'V\&A Digital Features' (Figure 7). Ten users were video and audio recorded while they were interacting with the application. We looked into their body posture, hands movement and gesture, and face expression. (Figure 8) Three researchers with different backgrounds looked into the videos and rated each user on their engagement level with the application on a scale from 1 to $5(1-$ not at all engaged, 2 - to a little extent engaged, 3 - neutral, 4 - to a moderate extent engaged, 5 - to a large extent engaged). We also took into account how much time the visitors spent with the installation, whether they had any interruptions and whether they were alone or in a group.
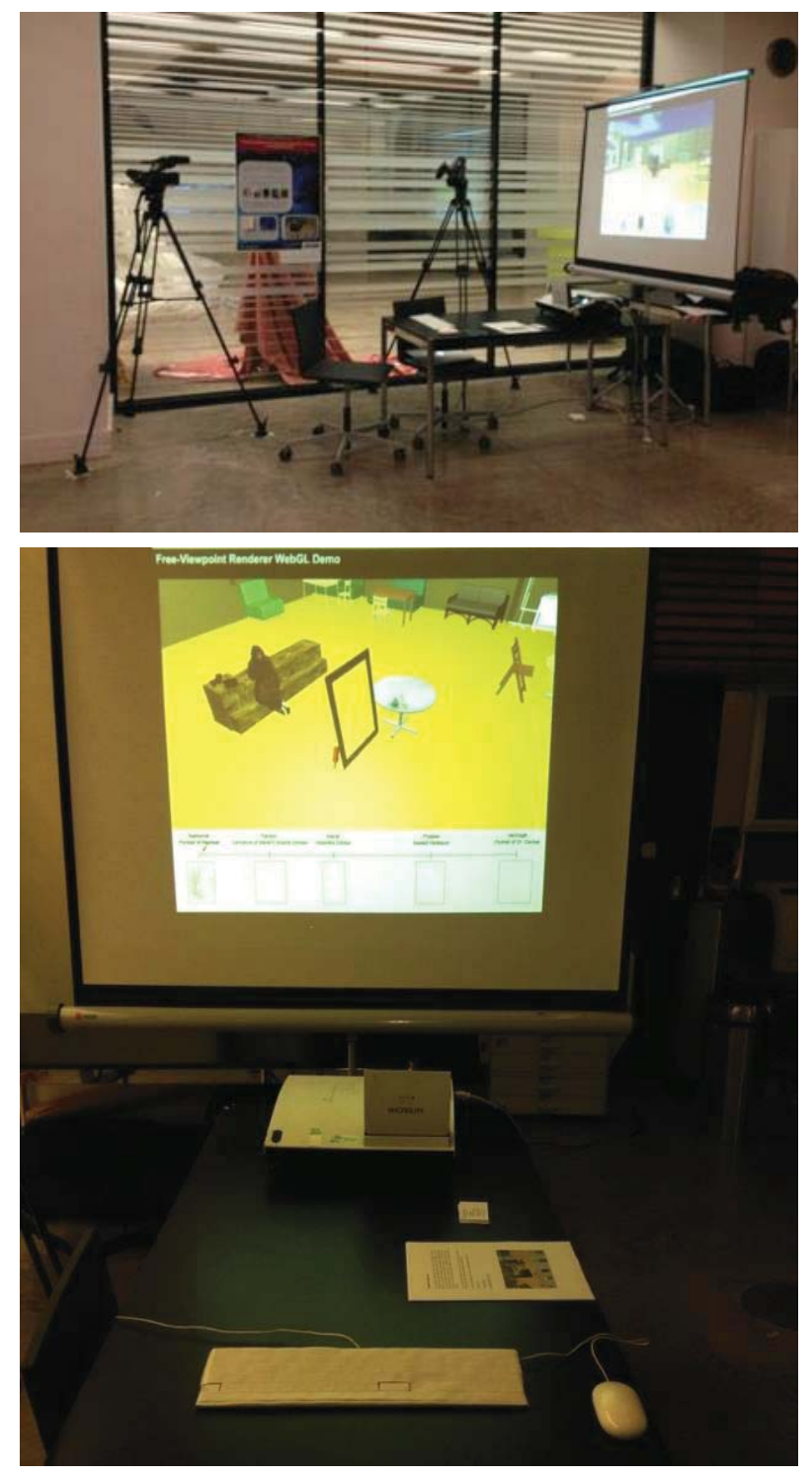

Figure 7: Setting of the installation in the Victoria and Albert Museum, London

\subsection{Results from the second study}

The ten visitors who participated in the study spent between 2 and 9 minutes interacting with the application. Half of the users did not have any interruptions while they were trying to find the correct view-point in the virtual environment, while the other half of the users got distracted between one and three times showing indications of less involvement in the performed activity. Three arbitrarily chosen independent experts were asked to rate the users' engagement according to three categories - body posture, hand movement and face expression. It was taken into account how people were sitting, whether they were occupying the whole seat or sitting on the edge of the chair. Another body feature examined was if people were leaning forward or backwards. It was also observed if users were using both their hands to interact with the system or not, and what kind of facial expression they had - smile, frown, intense eye stare or if were they often looking away from the screen.
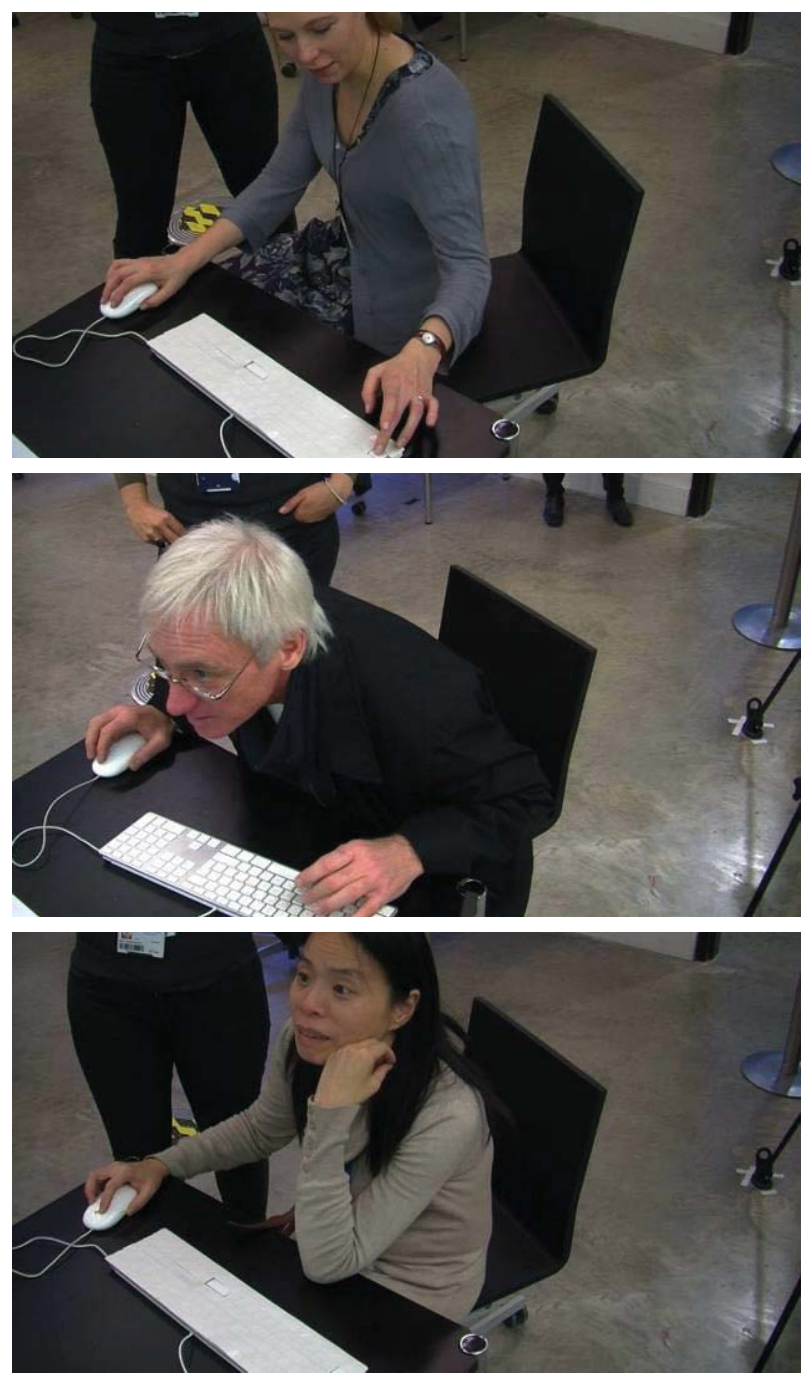

Figure 8: Visitors interacting with the application in the Victoria and Albert Museum, London 
The averaged ratings from all experts for body posture, hands movement and face expression were used to define the overall user engagement with the system as shown on Table 1 . The initial observations pointed out that the judgements of the three experts are not consistent, showing significant differences for some of the users.

Table 1: Body posture, hands movement, face expression and user engagement (averaged over the three expert ratings; one row per user)

\begin{tabular}{|c|c|c|c|}
\hline $\begin{array}{l}\text { Body } \\
\text { posture }\end{array}$ & $\begin{array}{l}\text { Hands } \\
\text { gestures }\end{array}$ & $\begin{array}{l}\text { Face } \\
\text { expression }\end{array}$ & $\begin{array}{c}\text { Engage- } \\
\text { ment }\end{array}$ \\
\hline 4 & 4 & 5 & 4 \\
\hline 4 & 3 & 4 & 4 \\
\hline 3 & 4 & 4 & 4 \\
\hline 3 & 3 & 4 & 3 \\
\hline 2 & 3 & 3 & 2 \\
\hline 2 & 2 & 2 & 2 \\
\hline 3 & 4 & 3 & 3 \\
\hline 4 & 3 & 4 & 4 \\
\hline 4 & 3 & 4 & 3 \\
\hline 3 & 2 & 2 & 2 \\
\hline
\end{tabular}

The ratings from the three experts were statistically analysed using Cohen's Kappa (Fleiss 2003), a standard statistic for determining the level of interannotator agreement between two experts for qualitative items. Cohen's Kappa takes on values between -1 and 1 (1 indicating perfect agreement between the raters, -1 indicating perfect disagreement and 0 indicating that any agreement is totally due to chance). Applying this scheme across pairs of the experts, the values were around 0 . Another method that we have used for evaluating the relationship between the raters was the Intra-Class Correlation Coefficient (ICC), which is applied to examine the reliability of the data (Fleiss 2003). This method can be implemented using three different models. In our research, we have applied this one by which randomly chosen independent experts evaluate each user's engagement based on behaviour characteristics: body posture, hand movement, and face expression. This approach allows better evaluation of the reliability of ratings by comparing the variability of different ratings of the same expert to the total variation across all ratings and all experts. For calculation of the ICC we have applied the ANOVA (ANalysis Of Variants) data analysis tool and for the data of body posture, hand movement and face expression have obtained values for ICC around 0.2 with $95 \%$ approximate confidence interval: lower $=0$, upper $=0.75$. These results indicate that the judgements of the experts have significant variations at some positions.

It is likely that the situation could be improved by increasing the number of independent experts, and/or by defining more specific characteristics to be evaluated in order to estimate users engagement. However, the high variability of these ratings, across all three measurement categories, is interesting in itself: measuring user engagement is a non-trivial task which needs consideration if reliable conclusions are to be drawn from studies such as these. This problem will be a focus of our research and evaluation studies in future.

\section{FUTURE WORK}

For the next phase of our research we are planning to perform controlled user studies. One study will look into how interactivity contributes towards successful communication of the curator's story about the presented paintings and the connection between them. For this purpose we will compare two applications presenting the same virtual gallery but with two different interaction approaches. In one case the user would be able to navigate freely in the virtual environment, while in the second scenario a person would be able to interact with the application only by starting and stopping a video of somebody else interacting and navigating with the virtual environment.

Furthermore, we intend to investigate the contribution of linear vs non-linear presentation of the narrative that connects the paintings to the successful communication of the information about the art and to the level of user engagement.

Another possible comparison would be between two applications with different user actions required to reveal the information in question - one where the information about the art is automatically displayed when the user virtually approaches the painting, and a second application in which the user has to perform a small task in order to be able to see more details about the displayed art.

\section{CONCLUSION}

New technologies such as Virtual Reality and freeviewpoint video provide us with exciting opportunities to present artworks in an interactive and more engaging way. The two projects presented here give us an example of how both technological and creative skillsets can be applied in the presentation of visual artworks in 3D interactive environments. 


\section{ACKNOWLEDGEMENTS}

Media and Arts Technology Programme, an RCUK Doctoral Training Centre in the Digital Economy.

BBC Research and Development Lab, Production Magic.

\section{REFERENCES}

Amicis, R., Girardi, G., Andreolli, M. and Conti, G. (2009) Game based technology to enhance the learning of history and cultural heritage, ACE '09: Proceedings of the International Conference on Advances in Computer Entertainment Technology, p. 451.

Bergamasco, M., Frisoli, A. and Barbagli, F. (2002) Haptics technologies and cultural heritage applications, Computer Animation, IEEE Proceedings of the Computer Animation (CA), pp. 25-32. IEEE.

Bovcon, N. (2008) Conceptual passages - Srečo Dragan's new media art projects, ELMAR, 50th IEEE International Symposium, vol. 2, pp. 483486.

Cavazza, M. and Mead, S. (2001) Virtual art galleries: a new kind of cultural objects?, Proceedings of International Conference on Image Processing, vol. 1, pp. 590-593. IEEE.

Eckert, M., Cubero, J.M., Sánchez, B., Martin, A. and Salgado, L. (2010) Low-cost VRML based architecture for adaptive and user customized virtual visits to art galleries, 3rd IEEE International Conference on Computer Science and Information Technology (ICCSIT), vol. 4, pp. 396-400. IEEE.

Fassbender, E., Wade, B., Carson, D. and Lea, T. (2010) Virtual galleries: First insights into the effect of the introduction of new media technologies, in art galleries, on economic and social wellbeing in urban and remote communities of the Northern Territory of Australia, 16th International Conference on Virtual Systems and Multimedia (VSMM), pp. 357-360. IEEE.

Fleiss, J. L., B. Levin, M. Cho Paik (2003) Statistical Methods for Rates \& Proportions, WileyInterscience.

Fortino, G., Confessore, G. and Mantuano, A. (2002) Design and implementation of a dynamic VRML-browsable, movie on-demand system distributed over Internet, ICME '02: Proceedings of IEEE International Conference on Multimedia and Expo, vol. 1, pp. 249-252. IEEE.

Graham, B. and Cook, S. (2010) Rethinking Curating. Art after New Media, The MIT Press, p. 283.
Hendricks, Z., Tangkuampien, J. and Malan k. (2003) Virtual galleries: Is 3D better? AFRIGRAPH '03: Proceedings of the 2nd International Conference on Computer Graphics, Virtual Reality, Visualisation and Interaction in Africa, pp. 17-24.

Hernandez, L., Taibo, J., Seoane, A., Lopez, R. and Lopez, R. (2003) The empty museum. Multiuser interaction in an immersive and physically walkable VR space, Proceedings of IEEE International Conference on Cyberworlds, pp. 446452. IEEE.

Hung, H. and Jacquemin C. (2010) Encounter (resonances), $M M$ '10: Proceedings of the International Conference on Multimedia, pp. 14211424

Maleshkova, J., Pansiot, J., and Grau, O. (2012) Through the painter's eye: Interactive 3D presentation of paintings on the web. In Proceedings of the Conference of Visual Media Production (CVMP 2012), p. 18. ACM Press.

Masa, M., Zara, J. and Smetana, P. (2003) eGaLab - a new dimension for artists, Proceedings of IEEE International Conference on Cyberworlds, pp. 466471. IEEE.

Minamizawa, K., Kakehi, Y., Nakatani,M., Mihara, S. and Tachi S. (2012) TECHTILE toolkit: a prototyping tool for designing haptic media, Proceedings of SIGGRAPH '12: Emerging Technologies. ACM Press.

Muller, A., Leissler, M., Hemmje, M. and Neuhold, E. (1999) Towards the Virtual Internet Gallery, Multimedia Computing and Systems, IEEE International Conference on Multimedia, vol. 2, pp. 214-219. IEEE.

O'Doherty, B. (1986) Inside the White Cube: The Ideology of the Gallery Space. University of California Press, p. 14.

Singh, G. (2011) Her own virtual gallery of art, Computer Graphics and Applications, vol. 31, issue 6, pp. 4-5. IEEE.

Ullrich, T. and Žára J. (2002) Interactive virtual gallery, SCCG '02: Proceedings of the $18^{\text {th }}$ Spring Conference on Computer Graphics, pp. 43-47.

Xiang Y. and Yam S. (2003) Embodied tour guide in an interactive virtual art gallery, Proceedings of IEEE International Conference on Cyberworlds, pp. 432-439. IEEE.

Zheng, J., Yuan, X. and Chee, Y. (2005) Designing multiparty interaction support in Elva, an embodied tour guide, AAMAS '05: Proceedings of the Fourth International Joint Conference on Autonomous Agents and Multi-Agent Systems, pp. 929-936. 